\title{
EYE LINES: A tool for studying visual perception of size and angle
}

\author{
WALTER K. BEAGLEY \\ Alma College, Alma, Michigan
}

\begin{abstract}
EYE LINES is a set of programs for preparing and running experiments that measure the perception of the size, position, or angle of orientation of visual stimuli. A drawing program builds images as collections of linked line-segment vectors. The experiments involve the method of adjustment. Subjects use a mouse to adjust the lengths or angles of specified line segments. Designed for use both in research and instructional laboratories, the programs run on the Macintosh.
\end{abstract}

A variety of microcomputer programs facilitate the display of images in perceptual or cognitive research (see, e.g., Meike, Bharucha, \& Baird, 1987). Most present images that consist of pixel mosaics. (These are often called bit-mapped graphics, or "paint" files.) Although such images can be displayed very quickly, they are not easy to modify or manipulate during experiments. The programs described here use "vector" graphics, in which the elements of an image are defined in terms of coordinates, lengths, angles, and links to other elements. Although such images take longer to draw on the screen, they can be manipulated in real time. Depending on which characteristic and which elements are made adjustable, shapes can be moved, resized, rotated, stretched, or distorted, and they can be used to implement any procedure that requires visually guided adjustment. The purpose of this project was to produce a system with which the method of adjustment could be used to study geometric illusions of size and form.

A primary design objective was that the system should have sufficient precision and flexibility for serious research, but, like a word processor, that it should have a useful subset of functions that a beginner could learn quickly. Having built an earlier, mainframe version that required the learning of numerous commands (Beagley, 1985), I decided to use Macintosh-style menus to shorten training time and make the system productive in an introductorylevel laboratory course. Finally, because of the heterogeneous computing environment now found at most institutions, I wanted it transportable between Macintosh and MS-DOS. It should be possible to draw images and set up an experiment on one machine but collect data with the other. This suggested that images, experiments, and data should be stored in plain text files that would be readable and editable with any computer. It also necessitated making some compromises with respect to the use of operating system features.

Correspondence and requests for information about acquiring EYE LINES should be addressed to Walter Beagley, Department of Psychology, Alma College, Alma, MI 48801. 20898WKB@MSU.Bitnet.

\section{The Programs}

The EYE LINES system consists of three programs. The first is used to construct images; the second, to set up experiments; and the third, to run experiments and store the data.

EL DRAW. The drawing program constructs images that are composed of line segments. The experimenter may use the mouse to draw lines and/or type coordinates into a spreadsheet that shares the screen with the image window. Regardless of how a segment is entered, its characteristics are arrayed across a single row of the spreadsheet. The first six columns contain the $x$ - and $y$-coordinates of the line's startpoint, the line's length and angle, and the $x$ - and $y$-coordinates of the line's endpoint (see Figure 1A). A seventh column contains the (optional) specifications of color and line width.

Segments can be linked together in several ways. To establish a link, the program ties the startpoint of a line to the start-, mid-, or endpoint of a previously existing segment. For example, in Figure 1A, Segments 2-5 do not have $x$ - and $y$-coordinates for their startpoints. Instead, Segment 2 shows an " $S$ " and a " 1 ," which indicates that it is to start wherever Segment 1 starts. Segment 4 shows an " $E$ " and a " 1 ," to indicate that it will start wherever Segment 1 ends. If Segment 1 is moved, lengthened, or rotated, all attached segments will move while they maintain these links. There is also a special "rubber" segment type, which may be linked to two other segments and which will stretch and/or rotate to maintain both connections as changes are made.

An image can be edited by making changes in the spreadsheet. A change in any segment will, of course, cause changes in any other segments that are linked to it. To make it easier to work back and forth between the image and the spreadsheet, both are displayed simultaneously, either can be temporarily hidden, and the spreadsheet window can be moved or resized so that it will not overlap with important parts of the image. There is also an option that can be used to cause on-off blinking of the segment associated with the spreadsheet row on which the cursor is placed. 
A. Image and Image File

Müller-Lyer

$\begin{array}{cllllll}\# & \text { SX } & Y & \text { LEN } & A & E X & Y \\ 1 & 2000 & 4500 & 4000 & 0 & 6000 & 4500 \\ 2 & S & 1 & 1000 & 150 & 1134 & 5000 \\ 3 & S & 1 & 1000 & 210 & 1134 & 4000 \\ 4 & E & 1 & 1000 & 30 & 6866 & 5000 \\ 5 & E & 1 & 1000 & 330 & 6866 & 4000 \\ 6 & 3000 & 3000 & 4000 & 0 & 7000 & 3000 \\ 7 & S & 6 & 1000 & 30 & 3866 & 3500 \\ 8 & \text { S } & 6 & 1000 & 330 & 3866 & 2500 \\ 9 & E & 6 & 1000 & 150 & 6134 & 3500 \\ 10 & E & 6 & 1000 & 210 & 6134 & 2500\end{array}$

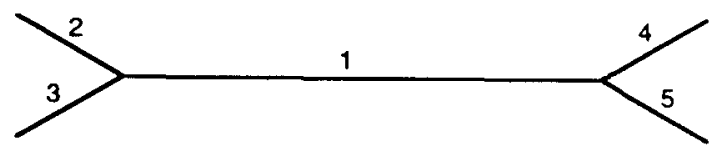

C

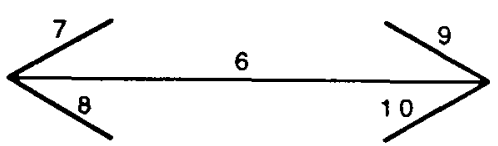

\section{B. Experiment Set-up}

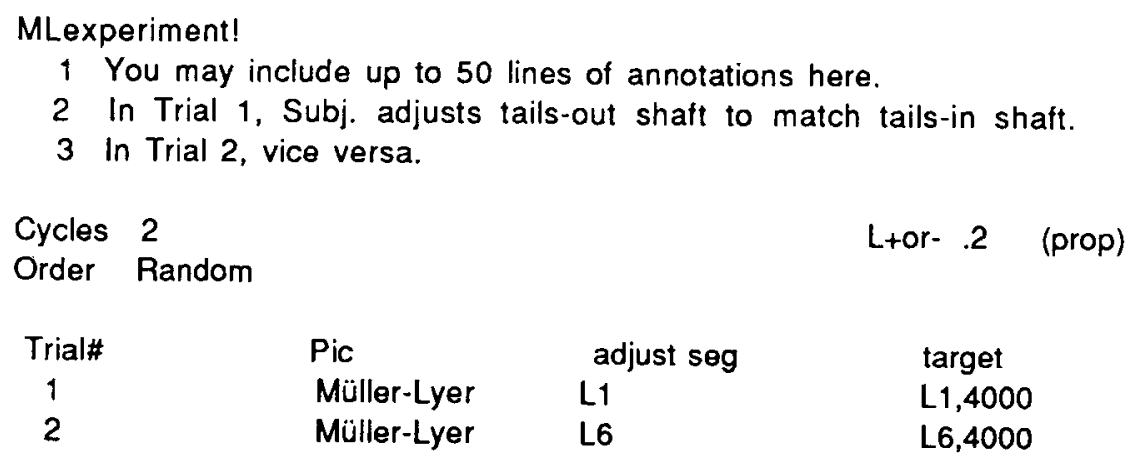

\section{Raw Data}

\begin{tabular}{|c|c|c|c|c|c|c|c|c|c|}
\hline Tr\# & time1-k & time2-k & Pic & seg & result & error & time3-k & time4-k & WB \\
\hline 1 & & & Müller-Lyer & 1 & 3262 & -738 & $8.68 \mathrm{~d}$ & & \\
\hline 2 & & & Müller-Lyer & 6 & 4622 & 622 & $13.98 \mathrm{~d}$ & & \\
\hline 2 & & & Mülier-Lyer & 6 & 4778 & 778 & $6.42 \mathrm{~d}$ & & \\
\hline 1 & & & Müller-Lyer & 1 & 3240 & -760 & $13.73 d$ & & \\
\hline Tr\# & timei-k & time2-k & Pic & seg & result & error & time3-k & time4-k & GJH \\
\hline 1 & & & Müiler-Lyer & 1 & 3375 & -625 & $6.08 d$ & & \\
\hline 2 & & & Mülier-Lyer & 6 & 4602 & 602 & $12.98 \mathrm{~d}$ & & \\
\hline 1 & & & Müller-Lyer & 1 & 3275 & - 725 & $7.50 d$ & & \\
\hline 2 & & & Müller-1_yer & 6 & 4733 & 733 & $8.00 \mathrm{~d}$ & & \\
\hline
\end{tabular}

Figure 1. Simple experiment showing sample output from each of the three programs. (A) EL DRAW. (B) EL SET. (C) EL RUN. 
Other features include simulation of monitor screens larger or smaller than the one currently in use, and the ability to export images for use with other graphics programs. Presently, arcs or circles must be constructed from short straight lines. This can be automated in the freehand mouse drawing mode. Text strings may be added to an image as stimulus elements or as labels or instructions. It is also possible to enter notes or annotations that are not displayed as part of the image.

EL SET. The set-up program displays another spreadsheet (see Figure 1B). For each trial, the experimenter enters the name of the image (column 1); what type of adjustment will occur ( $\mathrm{L}$ for length, $\mathrm{A}$ for angle), and which segment or segments are to be adjustable (column 2); and which of the adjustable segments is to be measured, as well as a target value to which it should be compared (column 3). For example, on Trial 1, the machine will display the image entitled "Müller-Lyer." The subject will adjust the length of Segment 1, after which the machine will record the resulting length of Segment 1 and compare it to its target value of 4,000 units.

The scrolling field above the spreadsheet will accept up to 50 lines of notes. There is also a set of choices that apply to the entire experiment: (1) cycles, each subject may be run through the trials as many times as desired; (2) order, trials may be run in fixed, random, or counterbalanced order; and (3) +or-, the initial length or angle of the adjustable segments can be set to vary by a random fraction of the entered value. In the example in Figure 1B, the horizontal shaft of the Müller-Lyer figure is set to vary by up to $20 \%$ of its nominal length, thus falling somewhere between 3,200 and 4,800 units. This can be used to control for psychophysical effects caused by direction of adjustment.

Although length and angle are currently the only available adjustment types, the position of an object can be made adjustable by linking the object to the end of an adjustable, invisible line. Shapes can be changed by adjusting some segments of an object but not others, or by lengthening some segments while shortening others. Finally, an advanced mode includes multiple field experiments, allows the collection of key input at several points during each trial, and provides additional choices that control the appearance and speed of image display.

EL RUN. The third program conducts the experiment and records the data. On each trial, the machine draws the image and then waits for a response. The subject can adjust the image by holding down the mouse button and moving the mouse up or down. When satisfied with the adjustment, the subject hits any key to advance to the next trial. The machine records: (1) result (adjusted value), (2) error (adjusted value minus target value), (3) elapsed time, and (4) the key that has been pressed to terminate the trial (see Figure 1C, which shows data for 2 subjects). An experiment may be temporarily suspended at any time so that the experimenter can look at data or enter comments. All data for a given experiment are accumulated in a single file.

\section{Limitations}

The maximum number of trials in an experiment and the maximum number of segments in an image depend on the amount of available memory. A basic Macintosh with $1 \mathrm{MB}$ of memory can handle 32 images, each with 50 segments. The number of segments that can be manipulated at once is limited by the time that is required to erase and redraw them. As the number increases, the blink between erase and redraw grows longer. On a Macintosh IIx in color, approximately 12 segments can be changed without the blinking's becoming bothersome.

The accuracy of length and the accuracy of angle are determined by the number of pixels per degree of visual angle and are thus affected by both monitor size and distance. Most monitors can easily be calibrated so that all lines of a specified length appear to be equal. Angle calibration is a bit more difficult. The geometry of most screens is not planar, but slightly distorted, especially near the edges. This requires proper experimental controls and careful placement of elements. The flat screens on portables might eliminate these concerns. Finally, the "stairsteps" produced by the scan lines make it impossible to do angle adjustment experiments with lines that are within $5^{\circ}$ of vertical or horizontal.

EYE LINES has not been designed to provide tachistoscopic accuracy. In tests run so far, timing was accurate to approximately one twentieth of a second, except when complex images were being drawn.

\section{Outcomes and Future Plans}

Undergraduates familiar with the Macintosh were able to run a prepared experiment with only $10 \mathrm{~min}$ of explanation. After half an hour of training, intermediate-level students were able to design their own experiments to investigate factors that influence the strength of MüllerLyer, Ponzo, and Zöllner illusions (though not without some false starts). Although it is too early to assess how productive EYE LINES will be as a research tool, we are currently using it to investigate interactions between angle contrast effects (Kurtenbach \& Magnussen, 1981) and illusions of length. Clearly, the system meets the objective of being usable by people with widely varying levels of expertise.

Note that the examples presented here do not define the boundary of potential uses. As an exploratory tool, EYE LINES quickly generates many variations on an image. This speeds the process of choosing optimal stimulus configurations. It also allows observation of dynamic or timedependent effects. We have used it to present a 3-D animation of the reversing Necker cube. It could be used to produce variations on the classic rod-and-frame test (Reinking, Goldstein, \& Houston, 1974). Finally, it need not be limited to perception research. For example, it could be used to implement graphics rating scales on which the subject would indicate the rating by adjusting the length of a bar or the rotation of a dial. Although the system was built primarily to run method-of-adjustment procedures, it is appropriate for any study in which either 
the experimenter or the subject has to change an image while the experiment is being run.

\section{REFERENCES}

BeAGLEY, W. K. (1985). Interaction of Müller-Lyer with filled-unfilled space illusion: An explanation of Müller-Lyer asymmetry. Perception \& Psychophysics, 37, 45-49.

Kurtenbach, W., \& Magnussen, S. (1981). Inhibition, disinhibition, and summation among orientation detectors in human vision. $E x$ perimental Brain Research, 43, 193-198.

Meike, B., Bharucha, J., \& Baird, J. (1987). MINDLAB [Computer program]. Hanover, NH: Dartmouth College, Courseware Development Group.

Reinking, R., Goldstein, G., \&ouston, B. (1974). Cognitive style, proprioceptive skills, task set, stress, and the rod-and-frame test of field orientation. Journal of Personality \& Social Psychology, 30 , 807-811. 